\title{
SeleçÃo de Espécies de Adubos Verdes Visando À FitorRemediação DE Diclosulam ${ }^{1}$
}

\author{
Selection of Green Manure Species aiming at Diclosulam Phytoremediation
}

\author{
MONQUERO, P.A. ${ }^{2}$, CÔRREA, M.C. ${ }^{3}$, BARBOSA, L.N. ${ }^{3}$, GUTIERREZ, A. ${ }^{3}$, ORZARI,.$^{3}{ }^{3}$ \\ HIRATA, A.C.S. ${ }^{4}$
}

\begin{abstract}
RESUMO - Espécies de adubos verdes tolerantes ao herbicida diclosulam podem ser utilizadas em rotação de culturas para diminuir o efeito fitotóxico subsequente desse herbicida em plantas sensiveis, como o girassol ou milho. Este estudo teve como objetivo avaliar a tolerância de adubos verdes ao diclosulam e a capacidade dessas plantas em diminuir o efeito fitotóxico do herbicida no bioindicador Helianthus annuus. Foram avaliadas, em casa de vegetação, três doses do herbicida diclosulam $(0,0,035$ e 0,070 kg i.a. ha-1) em pré-emergência dos adubos verdes Dolichos lablab, Cajanus cajan, Canavalia ensiformis, Crotalaria juncea, C. breviflora, C. spectabilis, Mucuna deeringiana, M. cinerea, M. aterrima, Lupinus albus, Helianthus annuus, Pennisetum glaucum, Avena strigosa, Raphanus sativus e Calopogonium muconoides. Entre estas espécies, C. cajan, C. ensiformis, $M$. cinerea e $M$. aterrima foram selecionadas como as mais tolerantes, sendo avaliadas no campo com o herbicida diclosulam nas doses de 0, 0,035 e $0,070 \mathrm{~kg}$ i.a. ha ${ }^{1}$, em esquema fatorial 4 x 3 e quatro repetições. A parte aérea dessas plantas foi coletada após 60 dias da emergência, sendo semeado $H$. annuus como bioindicador do herbicida diclosulam. Os resultados evidenciaram C. cajan como a espécie mais promissora em diminuir o efeito fitotóxico do diclosulam em culturas agricolas sensiveis.
\end{abstract}

Palavras-chave: adubos verdes, impacto ambiental, descontaminação, seleção de plantas, agroquímicos.

\begin{abstract}
The tolerance of green manure species to diclosulam can be used in crop rotation schemes aiming to reduce the subsequent phytotoxic effect of this herbicide on sensitive plants, such as sunflower or corn plants. This study evaluated the tolerance of green manure to diclosulan and the capacity of these plants in reducing the phytotoxic effect of this herbicide on Helianthus annuus (sunflower). Three rates of diclosulam (0; 0.035 , and $0.070 \mathrm{~kg}$ a.i. ha-1) were evaluated under greenhouse conditions in pre-emergence of the following green manures: Dolichos lablab, Cajanus cajan, Canavalis ensiformis, Crotalaria juncea, C. breviflora, C. spectabilis, Mucuna deeringiana, M. cinerea, M. aterrima, Lupinus albus, Helianthus annuus, Pennisetum glaucum, Avena strigosa, Raphanus sativus and Calopogonium muconoides. C. cajan, C. ensiformis, M. cinerea, and $\boldsymbol{M}$. aterrima were selected as the most tolerant ones, being evaluated in the field with diclosulan at the rates of $0,0.035$, and $0.070 \mathrm{~kg}^{\mathrm{a}}$.i. ha $\mathrm{h}^{-1}$ in a $4 \times 3$ factorial scheme, with four repetitions. The shoots of these plants were collected 60 days after emergence, and $\boldsymbol{H}$. annuus was sown as bio indicator of diclosulam. The results showed that C. cajan was the most promising species to reduce the phytotoxic effect of diclosulam on susceptible cultures.
\end{abstract}

Keywords: green manure, environmental impact, decontamination, plant selection, agrochemicals.

Recebido para publicação em 27.8.2011 e aprovado em 10.1.2012

2 Professora Adjunta, Centro de Ciências Agrárias, Universidade Federal de São Carlos - CCA/UFSCar, Rodovia Anhanguera, Km 174, Araras, São Paulo, <pamonque@cca.ufscar.br>; ${ }^{3}$ Alunos de graduação do curso de Engenharia Agronômica, CCA/UFSCar, $<$ mauriciocorrea@hotmail.com>, <lincon_ngs@hotmail.com>, <alinebgutierrez@gmail.com>, <bela.orzari@hotmail.com>; ${ }^{4}$ Pesquisadora, APTA/Polo Regional da Alta Sorocabana, <andreiacs@apta.sp.gov.br> 


\section{INTRODUÇÃO}

As plantas daninhas são alvo de controle, pois podem interferir nas plantas cultivadas diretamente (liberação de compostos alelopáticos e competição por água, luz e nutrientes) ou indiretamente (hospedando pragas, interferindo na colheita ou na qualidade do produto agrícola, entre outros), embora muitas vezes essas plantas sejam refúgios e fonte de alimentação para insetos predadores de importantes pragas dos cultivos agrícolas (Pitelli, 1985).

O diclosulam é um herbicida do grupo químico triazolo pirimidina sulfonanilidas, indicado para controle de dicotiledôneas em pré-semeadura incorporada ou em pré-emergência na cultura de soja. Esse composto atua inibindo a enzima acetolactato sintase (ALS), a qual é essencial para a síntese de aminoácidos valina, leucina e isoleucina (Rodrigues \& Almeida, 2011).

O diclosulam e seus metabólitos apresentam valores de partição $(\mathrm{Kd})$ bastante baixos, o que demonstra potencial de movimentação vertical, facilitada pela solubilidade em água (124 ppm a pH 7,0 e 117 ppm a pH 5,0), constante de ionização ácido (pKa) de 4,09 e coeficiente octanol/água (Kow) de 1,42 (Yoder et al., 2000). O comportamento desse herbicida é fortemente influenciado pelos teores de umidade e matéria orgânica do solo; a degradação é microbiana, e a fotodegradação e volatilização são insignificantes (Rodrigues \& Almeida, 2011). A meia-vida do diclosulam é de 60 a 90 dias, dependendo das condições de clima e solo (Lavorenti et al., 2003). A recomendação é de que logo após a colheita da soja, em áreas tratadas com diclosulam, não sejam semeadas culturas como milho, girassol, sorgo e brássicas (Rodrigues \& Almeida, 2011). De acordo com Yoder et al. (2000), esse herbicida, aplicado na soja, pode causar injúrias em culturas subsequentes, como o milho. Conforme Brighenti et al. (2002), o diclosulam causou redução total no estande de girassol quando semeado 75 e 90 dias depois da aplicação do herbicida.

A fitorremediação envolve o emprego de plantas, de sua microbiota associada e de amenizantes (corretivos, fertilizantes, matéria orgânica etc.) do solo, além de práticas agronômicas que, se aplicadas em conjunto, removem, imobilizam ou tornam os contaminantes inofensivos ao ecossistema (Accioly $\&$ Siqueira, 2000). Essa técnica pode ser uma alternativa para reduzir o efeito de herbicidas em culturas sucedâneas suscetíveis (Pires et al., 2003). A planta utilizada com esse objetivo deve apresentar porte alto, alta produção de biomassa, rápido crescimento, fácil controle e sistema radicular profundo e vigoroso (Accioly \& Siqueira, 2000). A capacidade transpiratória elevada - visando ao carreamento do composto tóxico para a parte aérea e, ainda, sua consequente metabolização ou acúmulo nos tecidos - também é uma característica importante (Vose et al., 2000). Plantas que apresentam maior excreção de exsudatos contribuem para o aumento da densidade de microrganismos próximos às raízes, o que pode estimular a degradação dos produtos químicos.

Os adubos verdes são culturas geralmente muito competitivas com as plantas daninhas e vêm sendo alvo de estudo sobre remediação de herbicidas em áreas agrícolas (Pires et al., 2003; Santos et al., 2004a,b; Pires et al., 2005a,b; Procópio et al., 2005). O objetivo principal da utilização da adubação verde é a melhoria das propriedades fisicas e químicas do solo; entretanto, muitas dessas plantas possuem grande poder inibitório sobre determinadas plantas daninhas, mesmo após o corte e formação de uma cobertura morta sobre o solo. A cobertura morta protege o solo da radiação solar, dissipa a energia do impacto das gotas de chuva, reduz a evaporação de água e aumenta a eficiência da ciclagem dos nutrientes (Mateus et al., 2004), além de ser uma alternativa para o controle de plantas daninhas (Vidal \& Bauman, 1996; Severino \& Christoffoleti, 2001; Oliveira et al., 2002). O controle de plantas daninhas pela cobertura vegetal pode ocorrer tanto pelo efeito físico como pelos efeitos alelopáticos e biológicos (Theisen et al., 2000; Favero et al., 2001; Pitelli \& Pitelli, 2004).

A espécie $M$. aterrima, além de tolerar concentrações elevadas de trifloxysulfuronsodium e tebuthiuron, possui a qualidade de adubo verde largamente utilizado na rotação de culturas, consorciação com gramíneas para pastagens, além da capacidade da fixação de nitrogênio atmosférico pela associação com 
bactérias diazotróficas. Com a aplicação da fitorremediação, pode-se reduzir significativamente o intervalo entre a aplicação desses herbicidas e a semeadura de espécie cultivada sensivel à presença dessas moléculas no solo.

O objetivo deste trabalho foi selecionar espécies de adubos verdes tolerantes ao diclosulam e com potencial de redução do efeito fitotóxico desse herbicida na cultura do girassol.

\section{MATERIAL E MÉTODOS}

No experimento I, em casa de vegetação, foram avaliadas 15 espécies de adubos verdes quanto à tolerância ao herbicida diclosulam. As espécies avaliadas foram Dolichos lablab, Cajanus cajan, Canavalia ensiformis, Crotalaria juncea, C. breviflora, C. spectabilis, Mucuna deeringiana, $M$. cinerea, $M$. aterrima, Lupinus albus, Helianthus annuus, Pennisetum glaucum, Avena strigosa, Raphanus sativus e Calopogonium muconoides. O delineamento foi em blocos ao acaso, com três repetições. Os tratamentos foram dispostos em esquema fatorial $15 \times 3$, sendo constituídos pelas espécies vegetais e doses de diclosulam $(0,0,035$ e 0,070 kg i.a. ha ${ }^{-1}$ ).

As sementes das espécies $M$. deeringiana, $M$. cinerea, $M$. aterrima e $C$. ensiformis tiveram sua dormência quebrada por meio de escarificação mecânica, com uso de uma lixa. Após a semeadura das espécies, os vasos foram irrigados de forma a manter a umidade do solo em torno de $80 \%$ até o final de avaliação. O monitoramento foi feito pelo método não destrutivo TDR (Time Domain Reflectometry).

O solo utilizado no experimento foi classificado como Latossolo Vermelho Distroférrico (Embrapa, 1997), proveniente da camada de 0-20 cm, sem histórico de uso de herbicidas. As análises química e física apresentaram as seguintes características: $\mathrm{pH}$ em $\mathrm{CaCl}_{2}$ de 6,2; $36 \mathrm{~g} \mathrm{dm}^{-3}$ de MO; $14 \mathrm{mg} \mathrm{dm}^{-3}$ de P; $2,4 \mathrm{mmol}_{\mathrm{c}} \mathrm{dm}^{-3}$ de $\mathrm{K} ; 29 \mathrm{mmol}_{\mathrm{c}} \mathrm{dm}^{-3}$ de $\mathrm{Ca}$; $13 \mathrm{mmol}_{\mathrm{c}} \mathrm{dm}^{-3} \mathrm{de} \mathrm{Mg}$; $0 \mathrm{mmol}_{\mathrm{c}} \mathrm{dm}^{-3} \mathrm{de} \mathrm{H} \mathrm{+} \mathrm{Al;}$ 44,4 $\mathrm{mmol}_{\mathrm{c}} \mathrm{dm}^{-3} \mathrm{de} \mathrm{SB}$; 68,4 $\mathrm{mmol}_{\mathrm{c}} \mathrm{dm}^{-3} \mathrm{de}$ CTC; $64 \%$ de saturação por bases; $560 \mathrm{~g} \mathrm{~kg}^{-1}$ de argila; $240 \mathrm{~g} \mathrm{~kg}^{-1}$ de silte; e $200 \mathrm{~g} \mathrm{~kg}^{-1}$ de areia.

A unidade experimental foi um vaso de polietileno contendo $20 \mathrm{~L}$ de solo, recoberto internamente com saco plástico para impedir a lixiviação do herbicida. O diclosulam foi aplicado no solo contido nos vasos em préemergência das espécies vegetais. $\mathrm{Na}$ aplicação do herbicida foi utilizado um pulverizador costal pressurizado com $\mathrm{CO}_{2}$, provido de barra de pulverização contendo dois bicos tipo leque Teejet 110.02 e com volume de aplicação de $200 \mathrm{~L} \mathrm{ha}^{-1}$. A umidade relativa do ar, medida no início da aplicação, a temperatura do ar e a velocidade do vento foram de $72 \%, 24,8{ }^{\circ} \mathrm{C}$ e $4,0 \mathrm{~km} \mathrm{~h}^{-1}$, respectivamente. Todas as espécies foram semeadas um dia após a aplicação do herbicida, sendo utilizadas cinco sementes de cada espécie por vaso.

A fitotoxicidade do herbicida nas plantas foi avaliada aos 15, 30 e 45 dias após a semeadura (DAS). Aos 46 DAS também foi avaliada a massa de matéria seca da parte aérea e das raízes das espécies vegetais.

Para avaliação da fitotoxicidade do herbicida, foram atribuídas notas de 0 a 100, em que 0 representou ausência de sintomas e 100 a morte da planta (ALAM, 1974). Para determinar a massa de matéria seca da parte aérea e das raízes das espécies vegetais, o material foi seco em estufa com circulação de ar forçada $\left(60 \pm 2{ }^{\circ} \mathrm{C}\right)$ por 72 horas. Os dados foram submetidos à análise de variância, sendo as médias significativas comparadas pelo teste de Tukey a $5 \%$ de probabilidade.

O experimento 2 foi conduzido em campo, sendo avaliadas as espécies C. cajan, $C$. ensiformis, $M$. cinerea e $M$. aterrima, consideradas tolerantes ao diclosulam de acordo com o experimento 1, e três doses do herbicida diclosulam $(0,0,035$ e 0,070 kg i.a. ha-1), em fatorial $4 \times 3$.

A unidade experimental foi composta por parcela medindo $8 \mathrm{~m}^{2}$, em área sem histórico de uso de herbicidas. O delineamento utilizado foi o de blocos ao acaso, com quatro repetições. As análises química e física do solo apresentaram as seguintes características: $\mathrm{pH}$ em $\mathrm{CaCl}_{2}$ de 6,0; $25 \mathrm{~g} \mathrm{dm}^{-3}$ de MO; $7 \mathrm{mg} \mathrm{dm}^{-3}$ de P; 0,9 $\mathrm{mmol}_{\mathrm{c}} \mathrm{dm}^{-3}$ de $\mathrm{K} ; 43 \mathrm{mmol}_{\mathrm{c}} \mathrm{dm}^{-3} \mathrm{de} \mathrm{Ca}$; $20 \mathrm{mmol}_{\mathrm{c}} \mathrm{dm}^{-3} \mathrm{de} \mathrm{Mg}$; $0 \mathrm{mmol}_{\mathrm{c}} \mathrm{dm}^{-3} \mathrm{de} \mathrm{H}+\mathrm{Al}$; $63,9 \mathrm{mmol}_{\mathrm{c}} \mathrm{dm}^{-3}$ de SB; $77,9 \mathrm{mmol}_{\mathrm{c}} \mathrm{dm}^{-3} \mathrm{de}$ CTC; $82 \%$ de saturação por bases; $530 \mathrm{~g} \mathrm{~kg}^{-1}$ de argila; $310 \mathrm{~g} \mathrm{~kg}^{-1}$ de silte; e $160 \mathrm{~g} \mathrm{~kg}^{-1}$ de areia.

Planta Daninha, Viçosa-MG, v. 31, n. 1, p. 127-135, 2013 
O herbicida diclosulam foi aplicado com pulverizador costal, pressurizado com $\mathrm{CO}_{2}$, provido de barra de pulverização contendo dois bicos tipo leque Teejet 110.02 e com volume de aplicação de $200 \mathrm{~L} \mathrm{ha}^{-1}$. Por ocasião da aplicação, no período da manhã, o céu estava claro e a velocidade do vento era inferior a $4,0 \mathrm{~km} \mathrm{~h}^{-1}$. A temperatura média do ar e a umidade foram de $25,9{ }^{\circ} \mathrm{C}$ e $67 \%$, respectivamente.

A semeadura das espécies fitorremediadoras foi feita um dia após a aplicação do herbicida, com cinco sementes de cada espécie de adubo verde por metro linear. Após a semeadura das espécies, as parcelas foram irrigadas, quando necessário, com lâmina de $20 \mathrm{~mm}$ de água/dia, de forma a manter a umidade do solo, durante o período de avaliação. As temperaturas mínimas, máximas e médias durante a condução do experimento foram de $18,4,30,6$ e $24{ }^{\circ} \mathrm{C}$, e a precipitação pluvial média, de $30 \mathrm{~mm}$.

A parte aérea das espécies de adubo verde foi coletada aos 60 dias após a emergência, sendo, em seguida, semeado girassol (10 sementes por metro linear), espécie sensível ao diclosulam. Após 45 DAS do girassol, foram avaliados a altura das plantas, a biomassa seca da parte aérea, os sintomas de toxicidade nas plantas e a área foliar (AF). A altura das plantas foi medida do colo até o meristema apical. Para determinar a massa seca da parte aérea, o material colhido foi seco em estufa com circulação forçada de ar $\left(70 \pm 2{ }^{\circ} \mathrm{C}\right)$ por 72 horas. Para a avaliação da fitotoxicidade, foram atribuídas notas de 0 a 100, de acordo com os sintomas de intoxicação observados na parte aérea das plantas, em que 0 representava ausência de sintomas e 100 a morte da planta (ALAM, 1974). A área foliar foi medida utilizando-se o aparelho portátil Li-3000C. Os dados foram submetidos à análise de variância e de regressão linear (Zar, 1999). As curvas de regressão foram ajustadas utilizando-se o aplicativo Sigmaplot.

\section{RESULTADOS E DISCUSSÃO}

\section{Experimento 1}

Aos 15 DAS, na maior dose do herbicida, as maiores porcentagens de fitotoxicidade foram verificadas nas espécies $R$. sativus $(86,67 \%)$ e P. glaucum $(83,33 \%)$. D. lablab $(66,67 \%), M$. deeringiana (66,67\%), $M$. aterrima $(66,67 \%), H$. annus $(63,33 \%)$ e $C$. muconoides $(66,67 \%)$ apresentaram fitotoxicidade intermediária. As espécies menos suscetíveis foram C. cajan (20,0\%), C. ensiformis $(20,0 \%)$, C. breviflora $(20,0 \%)$ e L. albus $(30,0 \%)$ (Tabela 1).

Aos 30 DAS, a maior tolerância para diclosulam continuou sendo verificada em C. ensiformis $(16,67 \%)$, seguida por C. cajan $(33,33 \%)$ e $L$. albus $(36,67 \%)$. Houve aumento dos sintomas de fitotoxicidade em $C$. breviflora, de $20,0 \%$ para $95,0 \%$, dos 15 para os 30 DAS. $R$. sativus apresentou, na maior dose, $86,67 \%$ de fitotoxicidade aos 15 DAS e $100 \%$ aos 30 DAS. Comparando as doses de 0,035 e $0,070 \mathrm{~kg}$ i.a. ha ${ }^{-1}$ de diclosulam para cada espécie estudada, verifica-se diferença estatística para $M$. deeringiana, $M$. aterrima, $P$. glaucum, A. strigosa e C. muconoides (Tabela 1).

A porcentagem de fitotoxicidade nos adubos verdes, avaliada aos 45 DAS, evidenciou alta suscetibilidade das espécies L. alpus, $H$. annuus e $R$. sativus ao herbicida diclosulam, com 100\% de controle nas duas doses avaliadas. As espécies C. ensiformis e C. cajan foram as mais tolerantes ao efeito de diclosulam, mesmo na maior dose, com 10,0 e $13,3 \%$ de controle, respectivamente; $C$. ensiformis não diferiu da testemunha sem herbicida. Para $M$. aterrima e $M$. cinerea, observou-se efeito fitotóxico apenas na maior dose do diclosulam $(33,0$ e $36,0 \%$, respectivamente), com clorose nas folhas mais jovens. Comparando as duas doses estudadas, verificase que para a maioria das espécies não houve diferença significativa em relação à fitotoxicidade, apenas diferenças com relação à testemunha. Todavia, para D. lablab, C. breviflora, $M$. deeringiana, P. glaucum e C. muconoides, houve diferença entre as doses avaliadas; a maior dose causou maiores injúrias às plantas (Tabela 1). Esse composto atua inibindo a enzima acetolactato sintase (ALS), a qual é essencial para a síntese dos aminoácidos valina, leucina e isoleucina. Apresenta amplo espectro de controle como latifolicida e também pode promover a supressão do crescimento de algumas gramineas e ciperáceas (Rodrigues \& Almeida, 2011). 
Tabela 1 - Porcentagem de fitotoxicidade de diclosulam em espécies de adubos verdes, avaliada aos 15, 30 e 45 dias após a semeadura (DAS)

\begin{tabular}{|c|c|c|c|c|c|c|c|c|c|}
\hline \multirow{4}{*}{ Espécie } & \multicolumn{9}{|c|}{ Dias após semeadura (DAS) } \\
\hline & \multicolumn{3}{|c|}{15} & \multicolumn{3}{|c|}{30} & \multicolumn{3}{|c|}{45} \\
\hline & \multicolumn{9}{|c|}{ Doses do diclosulam (kg i.a. ha $^{-1}$ ) } \\
\hline & 0,0 & 0,035 & 0,070 & 0,0 & 0,035 & 0,070 & 0,0 & 0,035 & 0,070 \\
\hline Dolichos lablab & $0,00 \mathrm{aC}$ & $50,00 \mathrm{bcB}$ & $66,67 \mathrm{abA}$ & $0,00 \mathrm{aC}$ & $73,33 \mathrm{cA}$ & $83,33 \mathrm{bcA}$ & $0,00 \mathrm{aC}$ & $60,00 \mathrm{cB}$ & $85,00 \mathrm{bA}$ \\
\hline Cajanus cajan & $0,00 \mathrm{aC}$ & $10,00 \mathrm{fB}$ & $20,00 \mathrm{de} A$ & $0,00 \mathrm{aB}$ & $20,00 \mathrm{ijAB}$ & $33,33 \mathrm{eA}$ & $0,00 \mathrm{aB}$ & 16,67 efA & $13,33 \mathrm{hA}$ \\
\hline Crotalaria ensiformis & $0,00 \mathrm{aB}$ & $0,00 \mathrm{gB}$ & $20,00 \operatorname{de} A$ & $0,00 \mathrm{aA}$ & $10,00 \mathrm{jA}$ & $16,67 \mathrm{fA}$ & $0,00 \mathrm{aA}$ & $6,67 \mathrm{fA}$ & $10,00 \mathrm{hA}$ \\
\hline Crotalaria juncea & $0,00 \mathrm{aC}$ & $25,00 \mathrm{deB}$ & $53,33 \mathrm{cA}$ & $0,00 \mathrm{aB}$ & $40,00 \mathrm{ghA}$ & $45,00 \mathrm{de} A$ & $0,00 \mathrm{aB}$ & $40,00 \mathrm{dA}$ & $46,67 \mathrm{fgA}$ \\
\hline Crotalaria breviflora & $0,00 \mathrm{aA}$ & $0,00 \mathrm{gA}$ & $20,00 \mathrm{deA}$ & $0,00 \mathrm{aB}$ & $88,00 \mathrm{abA}$ & $95,00 \mathrm{aA}$ & $0,00 \mathrm{aC}$ & $88,33 \mathrm{abB}$ & $98,33 \mathrm{aA}$ \\
\hline Crotalaria spectabilis & $0,00 \mathrm{aC}$ & $23,33 \mathrm{eB}$ & $43,33 \mathrm{cdA}$ & $0,00 \mathrm{aB}$ & $53,33 \mathrm{fgA}$ & $40,00 \mathrm{eA}$ & $0,00 \mathrm{aB}$ & $66,67 \mathrm{cA}$ & $66,67 \mathrm{de} A$ \\
\hline Mucuna deeringiana & $0,00 \mathrm{aC}$ & $36,67 \mathrm{~dB}$ & $66,67 \mathrm{abA}$ & $0,00 \mathrm{aC}$ & 60,00 efB & $80,00 \mathrm{bcA}$ & $0,00 \mathrm{aB}$ & $36,67 \mathrm{dAB}$ & 60,00 efA \\
\hline Mucuna cinerea & $0,00 \mathrm{aB}$ & $43,33 \mathrm{cdA}$ & $56,67 \mathrm{bcA}$ & $0,00 \mathrm{aB}$ & $40,00 \mathrm{ghA}$ & $45,00 \mathrm{de} A$ & $0,00 \mathrm{aB}$ & $33,33 \mathrm{dA}$ & $36,00 \mathrm{gA}$ \\
\hline Mucuna aterrima & $0,00 \mathrm{aB}$ & 63,33 abcA & $66,67 \mathrm{abA}$ & $0,00 \mathrm{aC}$ & $36,67 \mathrm{hB}$ & $56,67 \mathrm{dA}$ & $0,00 \mathrm{aB}$ & $30,00 \mathrm{de} A$ & $33,00 \mathrm{gA}$ \\
\hline Lupinus albus & $0,00 \mathrm{aB}$ & $20,00 \mathrm{eA}$ & $30,00 \mathrm{dA}$ & $0,00 \mathrm{aB}$ & 30,00 hiA & $36,67 \mathrm{eA}$ & $0,00 \mathrm{aB}$ & $100,00 \mathrm{aA}$ & $100,00 \mathrm{aA}$ \\
\hline Helianthus annus & $0,00 \mathrm{aB}$ & $46,67 \mathrm{cA}$ & $63,33 \mathrm{bcA}$ & $0,00 \mathrm{aB}$ & $97,00 \mathrm{aA}$ & $100,00 \mathrm{aA}$ & $0,00 \mathrm{aB}$ & $100,00 \mathrm{aA}$ & $100,00 \mathrm{aA}$ \\
\hline Pennisitum glaucum & $0,00 \mathrm{aC}$ & $70,00 \mathrm{abB}$ & $83,33 \mathrm{aA}$ & $0,00 \mathrm{aB}$ & $83,33 \mathrm{bB}$ & $96,67 \mathrm{aA}$ & $0,00 \mathrm{aC}$ & $81,67 \mathrm{bB}$ & $100,00 \mathrm{aA}$ \\
\hline Avena strigosa & $0,00 \mathrm{aB}$ & $10,00 \mathrm{fB}$ & $50,00 \mathrm{cA}$ & $0,00 \mathrm{aC}$ & 53,33 efB & $86,67 \mathrm{abA}$ & $0,00 \mathrm{aB}$ & $96,67 \mathrm{aA}$ & $90,00 \mathrm{abA}$ \\
\hline Raphanus sativus & $0,00 \mathrm{aB}$ & $76,67 \mathrm{aA}$ & $86,67 \mathrm{aA}$ & $0,00 \mathrm{aB}$ & $96,67 \mathrm{aA}$ & $100,00 \mathrm{aA}$ & $0,00 \mathrm{aB}$ & $100,00 \mathrm{aA}$ & $100,00 \mathrm{aA}$ \\
\hline Calapogonium muconoides & $0,00 \mathrm{aC}$ & $40,00 \mathrm{cdB}$ & $66,67 \mathrm{abA}$ & $0,00 \mathrm{aB}$ & $65,00 \mathrm{de} A$ & $73,33 \mathrm{cA}$ & $0,00 \mathrm{aC}$ & $31,67 \mathrm{~dB}$ & $78,33 \mathrm{cdA}$ \\
\hline $\mathrm{CV}(\%)$ & & 25,81 & & & 11,20 & & & 12,27 & \\
\hline
\end{tabular}

Médias seguidas por letras iguais, maiúsculas na linha e minúsculas na coluna, não diferem estatisticamente pelo teste de Tukey a $5 \%$ de probabilidade.

Com relação à biomassa da parte aérea, observou-se que $C$. ensiformis apresentou maior acúmulo de biomassa $(12,47 \mathrm{~g})$, não havendo diferença significativa entre a menor dose $(11,40 \mathrm{~g})$ e a testemunha sem o herbicida (11,67 g). Quanto às demais espécies, os maiores acúmulos de biomassa seca da parte aérea no tratamento de $0,035 \mathrm{~kg}$ i.a ha ${ }^{-1}$ foram observados nas espécies $M$. aterrima $(8,33 \mathrm{~g})$ e M. cinerea (6,30 g) (Tabela 2). Alguns estudos indicam que $M$. aterrima e $C$. ensiformis são eficientes na descontaminação de áreas tratadas com os herbicidas trifloxysulfuronsodium e tebuthiuron, sendo a interação da fitoestimulação e fitodegradação o provável mecanismo envolvido na descontaminação (Procópio et al., 2005). Em se tratando de C. cajan, embora não tenham sido observadas diferenças significativas com relação aos tratamentos, o acúmulo de biomassa foi reduzido; a testemunha apresentou 1,52 g de biomassa seca. Ressalta-se que os resultados de acúmulo de massa seca são importantes, pois a escolha da espécie também deve considerar a taxa de crescimento e a produção de biomassa.
No tocante à biomassa seca das raízes das espécies de adubo verde, verifica-se que C. cajan, C. breviflora, C. spectabilis e C. muconoides não apresentaram diferenças significativas quanto ao desenvolvimento da raiz entre a testemunha e as doses avaliadas de diclosulam; entretanto, a maior produção de biomassa seca das raízes foi encontrada na espécie C. ensiformis, com 2,29 e 2,06 g nas doses de 0,035 e 0,070 kg i.a. ha-1 de diclosulam, respectivamente (Tabela 2). Essas informações são importantes, pois uma das características favoráveis aos fitorremediadores é o sistema radicular profundo e denso.

Com base nos resultados de porcentagem de fitotoxicidade e biomassa seca da parte aérea e raiz, as espécies C. ensiformis, $M$. aterrima, $M$. cinerea e C. cajan apresentaram maior tolerância ao herbicida diclosulam, sendo utilizadas no experimento 2 .

\section{Experimento 2}

Aos $45 \mathrm{DAS}$, na parcela previamente cultivada com C. ensiformis após a aplicação 
Tabela 2 - Biomassa seca (g por planta) da parte aérea e de raízes de espécies de adubos verdes sob doses do herbicida diclosulam, avaliada aos 45 DAS

\begin{tabular}{|l|c|c|c|c|c|c|}
\hline \multirow{2}{*}{ Espécie } & \multicolumn{3}{|c|}{ Biomassa $(\mathrm{g})$ da parte aérea } & \multicolumn{3}{c|}{ Biomassa $(\mathrm{g})$ das raízes } \\
\cline { 2 - 7 } & \multicolumn{5}{|c|}{ Doses de diclosulam $\left(\mathrm{kg}\right.$ i.a. ha $\left.{ }^{-1}\right)$} \\
\cline { 2 - 7 } & 0,0 & 0,035 & 0,070 & 0,0 & 0,035 & 0,070 \\
\hline Dolichos lablab & $1,51 \mathrm{fgA}$ & $1,40 \mathrm{efA}$ & $0,60 \mathrm{efgB}$ & $0,61 \mathrm{eA}$ & $0,39 \mathrm{efB}$ & $0,10 \mathrm{efC}$ \\
\hline Cajanus cajan & $1,52 \mathrm{fgA}$ & $1,32 \mathrm{fA}$ & $1,28 \mathrm{defA}$ & $0,29 \mathrm{fA}$ & $0,30 \mathrm{fA}$ & $0,30 \mathrm{dA}$ \\
\hline Crotalaria ensiformis & $11,67 \mathrm{aA}$ & $11,40 \mathrm{aA}$ & $12,47 \mathrm{aA}$ & $3,29 \mathrm{aA}$ & $2,29 \mathrm{aB}$ & $2,06 \mathrm{aC}$ \\
\hline Crotalaria juncea & $1,22 \mathrm{fghA}$ & $0,80 \mathrm{fgA}$ & $0,78 \mathrm{efgA}$ & $0,28 \mathrm{fAB}$ & $0,38 \mathrm{efA}$ & $0,26 \mathrm{deB}$ \\
\hline Crotalaria breviflora & $2,01 \mathrm{fA}$ & $0,02 \mathrm{gB}$ & $0,01 \mathrm{gB}$ & $0,08 \mathrm{gA}$ & $0,02 \mathrm{gA}$ & $0,01 \mathrm{fA}$ \\
\hline Crotalaria spectabilis & $0,44 \mathrm{hA}$ & $0,13 \mathrm{gB}$ & $0,09 \mathrm{gB}$ & $0,11 \mathrm{fgA}$ & $0,04 \mathrm{gB}$ & $0,03 \mathrm{fB}$ \\
\hline Mucuna deeringiana & $5,31 \mathrm{dA}$ & $4,43 \mathrm{cdB}$ & $1,83 \mathrm{dC}$ & $0,99 \mathrm{dA}$ & $0,55 \mathrm{deB}$ & $0,11 \mathrm{efC}$ \\
\hline Mucuna cinerea & $8,55 \mathrm{bA}$ & $6,30 \mathrm{cB}$ & $5,94 \mathrm{cB}$ & $1,83 \mathrm{bA}$ & $1,61 \mathrm{bB}$ & $1,35 \mathrm{bC}$ \\
\hline Mucuna aterrima & $7,33 \mathrm{cA}$ & $8,33 \mathrm{bA}$ & $7,67 \mathrm{abA}$ & $1,62 \mathrm{cA}$ & $1,11 \mathrm{cB}$ & $0,85 \mathrm{cC}$ \\
\hline Lupinus albus & $3,38 \mathrm{eA}$ & $2,36 \mathrm{~dB}$ & $1,49 \mathrm{deC}$ & $0,84 \mathrm{dA}$ & $0,62 \mathrm{~dB}$ & $0,42 \mathrm{dC}$ \\
\hline Helianthus annus & $0,57 \mathrm{ghA}$ & $0,02 \mathrm{gB}$ & $0,01 \mathrm{gB}$ & $0,15 \mathrm{fgA}$ & $0,01 \mathrm{gB}$ & $0,01 \mathrm{fB}$ \\
\hline Pennisitum glaucum & $0,50 \mathrm{hA}$ & $0,01 \mathrm{gB}$ & $0,01 \mathrm{gB}$ & $0,18 \mathrm{fgA}$ & $0,02 \mathrm{gB}$ & $0,01 \mathrm{fB}$ \\
\hline Avena strigosa & $0,31 \mathrm{hA}$ & $0,04 \mathrm{gB}$ & $0,02 \mathrm{gB}$ & $0,14 \mathrm{fgA}$ & $0,03 \mathrm{gB}$ & $0,01 \mathrm{fB}$ \\
\hline Raphanus sativus & $1,03 \mathrm{fghA}$ & $0,01 \mathrm{gB}$ & $0,01 \mathrm{gB}$ & $0,18 \mathrm{fgA}$ & $0,01 \mathrm{gB}$ & $0,01 \mathrm{fB}$ \\
\hline Calapogonium muconoides & $0,24 \mathrm{hA}$ & $0,16 \mathrm{fgA}$ & $0,19 \mathrm{fgA}$ & $0,08 \mathrm{gA}$ & $0,04 \mathrm{gA}$ & $0,06 \mathrm{fA}$ \\
\hline \multicolumn{1}{|c|}{ CV $(\%)$} & & 16,25 & & & 16,35 \\
\hline
\end{tabular}

Médias seguidas por letras iguais, maiúsculas na linha e minúsculas na coluna, não diferem estatisticamente pelo teste de Tukey a $5 \%$ de probabilidade.

do diclosulam, a maior dose do herbicida causou fitotoxicidade em $H$. annus acima de $80 \%$, o que é considerado alto, refletindo a baixa capacidade do adubo verde em fitorremediar o diclosulam na dose de 0,070 g i.a. ha-1 embora a espécie seja tolerante ao herbicida. Houve aumento da fitotoxicidade do herbicida com a elevação da dose e entre as avaliações (Tabela 3).

Com relação ao uso de C. cajan como fitorremediador, observou-se que aos 15 DAS do bioindicador houve atraso na germinação de $H$. annuus nas parcelas com diclosulam. Aos 45 DAS do bioindicador, entretanto, não houve diferença entre as doses do diclosulam: ambas apresentaram $65 \%$ de fitotoxicidade em H. annuus (Tabela 3).

Em relação à semeadura de $H$. annuus em área fitorremediada por $M$. cinerea, verificou-se fitotoxicidade no bioindicador de $80 \%$ na menor dose de diclosulam e de $100 \%$ na maior dose do herbicida. Portanto, embora $M$. cinerea tenha mostrado tolerância ao diclosulam, não apresentou fitorremediação do herbicida (Tabela 3).

No caso das parcelas com $M$. aterrima como fitorremediador, o girassol apresentou necrose nas parcelas com a dose maior de diclosulam, com $90 \%$ de fitotoxicidade. Na dose menor, as plantas se mostraram cloróticas e com atraso no desenvolvimento, com $70 \%$ de fitotoxicidade.

Foram observados menores acúmulos de biomassa em todas as parcelas que receberam a dose de 0,070 g i.a. ha ${ }^{-1}$ de diclosulam, principalmente nos fitorremediadores $M$. cinerea e $M$. aterrima. As parcelas que foram cultivadas com C. cajan e C. ensiformis como fitorremediadores apresentaram maior biomassa do girassol, inclusive na testemunha, corroborando os dados de fitotoxicidade (Figura 1A).

Verificou-se menor área foliar nas plantas de girassol com o uso do herbicida. O uso da espécie $M$. cinerea proporcionou menor área foliar do girassol na maior dose de diclosulam. Por outro lado, a maior área foliar de girassol foi precedida pelo uso de $C$. cajan como fitorremediador (Figura 1B).

O bioindicador cultivado após a retirada de $M$. cinerea e $M$. aterrima apresentou as menores alturas, corroborando as avaliações anteriores. A maior altura foi observada nas 
Seleção de espécies de adubos verdes visando à fitorremediação ...

Tabela 3 - Porcentagem de fitotoxicidade de H. annuus cultivada em sucessão a C. ensiformis, C. cajan, M. cinerea e M. aterrima, em área tratada com diclosulam em diferentes doses

\begin{tabular}{|c|c|c|c|c|c|c|c|c|c|}
\hline \multirow{4}{*}{ Espécie } & \multicolumn{9}{|c|}{ Fitotoxicidade (\%) } \\
\hline & \multicolumn{3}{|c|}{15 DAS } & \multicolumn{3}{|c|}{30 DAS } & \multicolumn{3}{|c|}{$45 \mathrm{DAS}$} \\
\hline & \multicolumn{9}{|c|}{ Doses do diclosulam (g i.a. ha $\left.{ }^{-1}\right)$} \\
\hline & 0,0 & 0,035 & 0,070 & 0,0 & 0,035 & 0,070 & 0,0 & 0,035 & 0,070 \\
\hline Canavalia ensiformis & $0,0 \mathrm{aB}$ & $0,0 \mathrm{bB}$ & $10,0 \mathrm{aA}$ & $0,0 \mathrm{aC}$ & $50,0 \mathrm{aB}$ & $80,0 \mathrm{aA}$ & $0,0 \mathrm{aC}$ & $65,0 \mathrm{bB}$ & $85,0 \mathrm{bA}$ \\
\hline Cajans cajan & $0,0 \mathrm{aB}$ & $5,0 \mathrm{abA}$ & $0,0 \mathrm{bB}$ & $0,0 \mathrm{aC}$ & $60,0 \mathrm{aB}$ & $80,0 \mathrm{aA}$ & $0,0 \mathrm{aB}$ & $65,0 \mathrm{bA}$ & $65,0 \mathrm{cA}$ \\
\hline Mucuna cinerea & $0,0 \mathrm{aB}$ & $3,33 \mathrm{abAB}$ & $5,0 \mathrm{aBA}$ & $0,0 \mathrm{aB}$ & $60,0 \mathrm{aA}$ & $70,0 \mathrm{aA}$ & $0,0 \mathrm{aC}$ & $80,0 \mathrm{aB}$ & $100,0 \mathrm{aA}$ \\
\hline Mucuna aterrima & $0,0 \mathrm{aB}$ & $8,33 \mathrm{aA}$ & $10,0 \mathrm{aA}$ & $0,0 \mathrm{aB}$ & $50,0 \mathrm{aA}$ & $50,0 \mathrm{bA}$ & $0,0 \mathrm{aC}$ & $70,0 \mathrm{abB}$ & $90,0 \mathrm{abA}$ \\
\hline $\mathrm{CV}(\%)$ & \multicolumn{3}{|c|}{59,28} & \multicolumn{3}{|c|}{14,70} & \multicolumn{3}{|c|}{11,17} \\
\hline
\end{tabular}

Médias seguidas por letras iguais, maiúsculas na linha e minúsculas na coluna, não diferem estatisticamente pelo teste de Tukey a $5 \%$ de probabilidade.
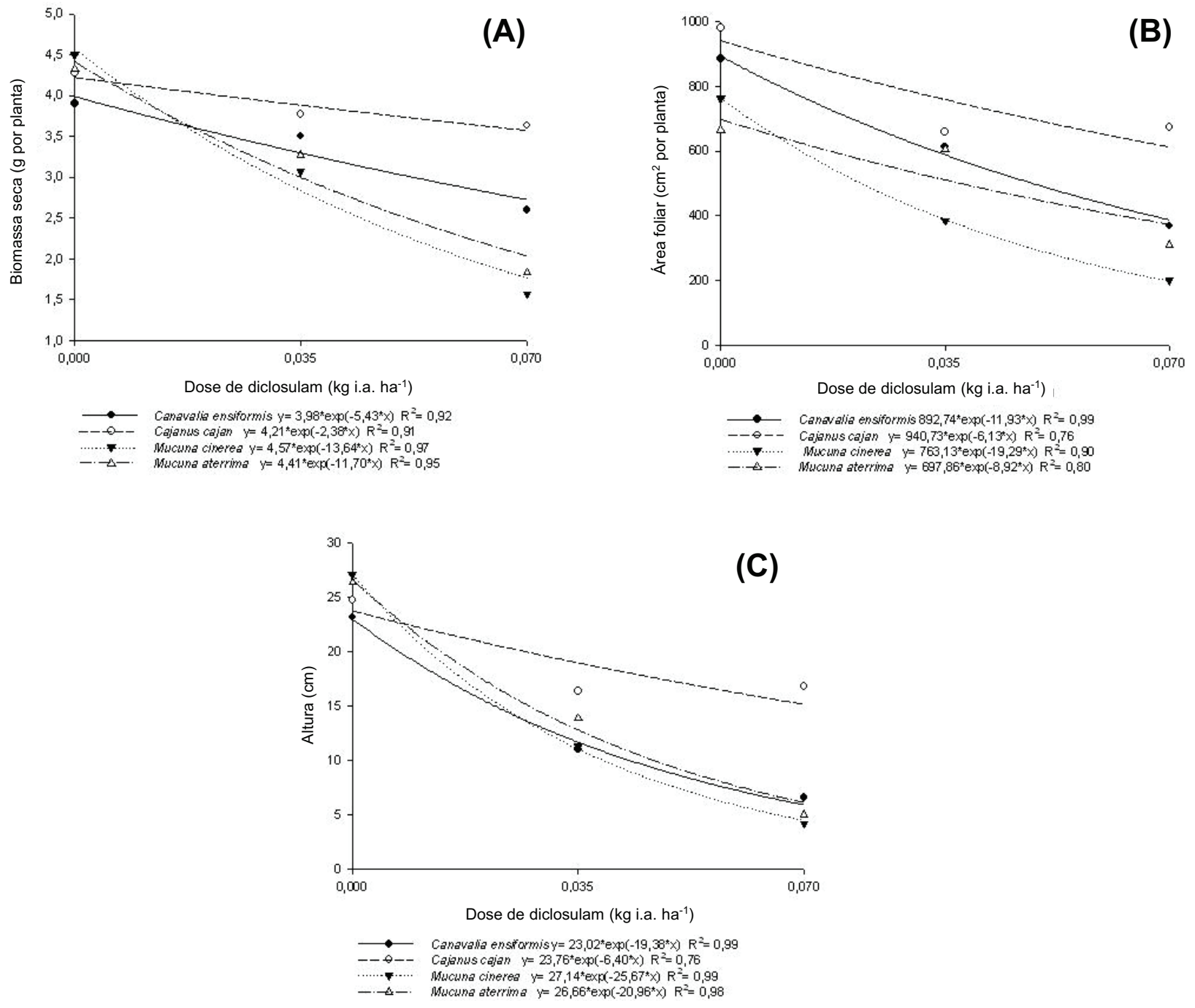

Figura 1 - Biomassa seca (A), área foliar (B) e altura (C) de plantas de girassol cultivado em sucessão aos adubos verdes C. ensiformis, C. cajan, M. cinerea e M. aterrima, em área tratada com diclosulam. 
parcelas que tiveram $C$. cajan como fitorremediador; na menor dose de diclosulam a altura do girassol foi de $15,10 \mathrm{~cm} \mathrm{e}$, na maior, de 19,40 cm, ainda assim diferindo da testemunha, que apresentou altura de $23,90 \mathrm{~cm}$. Quando o fitorremediador foi C. ensiformis, a altura do girassol na menor dose do herbicida foi de $11,75 \mathrm{~cm}$ e, na maior dose de diclosulam, de $5,93 \mathrm{~cm}$ (Figura 1C).

Existem pelo menos quatro mecanismos gerais que podem explicar a tolerância de uma planta a herbicidas: redução da concentração do herbicida no local de ação; absorção foliar e/ou translocação reduzida do herbicida; metabolização e/ou destoxificação intensa do herbicida a substâncias menos fitotóxicas; e perda de afinidade do herbicida pelo local de ação devido a uma alteração desse local, resultante de variabilidade genética (Sherman et al., 1996). Para que uma planta tolerante seja também eficiente como fitorremediadora, é importante que haja degradação da molécula herbicida; além disso, vale lembrar que na fitorremediação de orgânicos as plantas podem metabolizar os compostos, o que não quer dizer que eles serão completamente mineralizados. Em alguns casos, os metabólitos podem ser mais problemáticos do que os compostos originais (Pires et al., 2003).

Naturalmente, torna-se dificil reunir todas as características desejáveis de um fitorremediador em apenas uma espécie, porém aquela que for selecionada deve apresentar o maior número delas. Outro aspecto a ser observado é que, embora a maioria dos testes avalie plantas isoladas, várias espécies podem ser usadas em um mesmo local, ou ao mesmo tempo ou subsequentemente, para remover mais de um contaminante.

Os resultados permitem concluir que a espécie $C$. cajan é eficiente na remediação de solos contaminados com diclosulam.

\section{AGRADECIMENTOS}

À Fundação de Amparo à Pesquisa do Estado de São Paulo, pela bolsa de iniciação cientifica, e ao Grupo de Pesquisa em Ciências Agrárias da UFSCar, pelo apoio durante a condução do experimento.

\section{LITERATURA CITADA}

ACCIOLY, A. M. A.; SIQUEIRA, J. O. Contaminação química e biorremediação do solo. In: NOVAIS, R. F.; ALVAREZ V.; V. H.; SCHAEFER, C. E. G. R. Tópicos em Ciência do Solo. Viçosa-MG: Sociedade Brasileira de Ciência do Solo, 2000. v. 1. p. 299-352.

ASOCIATION LATINOAMERICANA DE MALEZAS ALAM. Recomendaciones sobre unificación de los sistemas de evaluación em ensayos de control de malezas. ALAM, v. 1, n. 1, p. 35-38, 1974.

BRIGHENTI, A. M. et al. Persistência e fitotoxicidade de herbicidas aplicados na soja sobre o girassol em

sucessão. Pesq. Agropec. Bras., v. 37, n. 4, p. 559-565, 2002.

EMPRESA BRASILEIRA DE PESQUISA

AGROPECUÁRIA - EMBRAPA. Centro Nacional de Pesquisa de Solos. Manual de métodos de análise de solo. 2.ed. Rio de Janeiro: 1997. 212 p.

FAVERO, C. et al. Modificações na população de plantas espontâneas na presença de adubos verdes. Pesq. Agropec. Bras., v. 36, n. 11, p. 1355-1362, 2001.

LAVORENTI, A. et al. Comportamento do diclosulam em amostras de um Latossolo Vermelho distroférrico sob plantio direto e convencional. R. Bras. Ci. Solo, v. 27, n. 2 ,

p. 183-190, 2003.

MATEUS, G. P.; CRUSCIOL, C. A. C.; NEGRISOLI, E. Palhada do sorgo de guiné-gigante no estabelecimento de plantas daninhas em área de plantio direto. Pesq. Agropec. Bras., v. 39, n. 6, p. 539-542, 2004

OLIVEIRA, T. K.; CARVALHO, G. J.; MORAES, R. N. S. Plantas de cobertura e seus efeitos sobre o feijoeiro em plantio direto. Pesq. Agropec. Bras., v. 37, n. 8, p. 1079-1087, 2002.

PERKOVICH, B. S. et al. Enhanced mineralization of [14C] atrazine in $K$. scoparia rhizosferic soil from a pesticidecontaminated site. Pestic. Sci., v. 46, n. 4, p. 391-396, 1996.

PIRES, F. R. et al. Seleção de plantas com potencial para fitorremediação de tebuthiuron. Planta Daninha, v. 21, n. 3, p. $451-458,2003$.

PIRES, F. R. et al. Fitorremediação de solos contaminados com tebuthiuron utilizando-se espécies cultivadas para adubação verde. Planta Daninha, v. 23, n. 4, p. 711-717. $2005 \mathrm{a}$

PIRES, F. R. et al. Inferências sobre atividade rizosférica de espécies com potencial para fitorremediação do herbicida tebuthiuron. R. Bras. Ci. Solo, v. 29, n. 4, p. 627-634, 2005 b. 
PITELLI, R. A. Interferência das plantas daninhas em culturas agrícolas. Inf. Agropec., v. 11, n. 129, p. 16-27, 1985.

PITELLI, R. A.; PITELLI, R. L. C. M. Biología e ecofisiologia das plantas daninhas. In: VARGAS, L.; ROMAM, E. S. (Ed.). Manual de manejo e controle de plantas daninhas. Bento Gonçalves: Embrapa Uva e Vinho, 2004. p. 29-56.

PROCÓPIO, S. O. et al. Fitorremediação de solo contaminado com trifloxysulfuron-sodium por mucuna-preta (Stizolobium aterrimum). Planta Daninha, v. 23, n. 4, p. 719-724, 2005.

RODRIGUES, B. N.; ALMEIDA, F. S. Guia de herbicidas. 6.ed. Londrina: Grafmarke, 2011. 697 p.

SANTOS, J. B. et al. Seletividade do herbicida trifloxysulfuron-sodium para fins de fitorremediação.

R. Ceres, v. 51, n. 1, p. 129-141, 2004a.

SANTOS, J. B. et al. Fitorremediação do herbicida trifloxysulfuron-sodium. Planta Daninha, v. 22, n. 2, p. 323-330, 2004b.

SEVERINO, F. J.; CRHISTOFFOLETI, P. J. Efeitos de quantidades de fitomassa de adubos verdes na supressão de plantas daninhas. Planta Daninha, v. 19, n. 2, p. 223-228, 2001.
VOSE, J. M. et al. Leaf water relations and sapflow in Eastern cottonwood (Populus detoides Bartr.) trees planted for phytoremediation of a groundwater pollutant. Inter. J. Phytorem., v. 2, n. 1, p. 53-73, 2000

SHERMAN, T. D.; VAUGHN, K. C.; DUKE, S. O.

Mechanism of action and resistance to herbicides. In: DUKE, S. O. (Ed.). Herbicides resistant crops. Boca Raton: CRC Press, 1996. p. 14-28.

THEISEN, G.; VIDAL, R. A.; FLECK, N. G. Redução da infestação de Brachiaria plantaginea em soja pela cobertura do solo com palha de aveia preta. Pesq. Agropec. Bras., v. 35, n. 4, p. 753-756, 2000.

VIDAL, R. A.; BAUMAN, T. T. Surface wheat (Triticum aestivum) residues, giant foxtail (Setaria faberi), and soybean (Glycine max) yield. Weed Sci., v. 44, n. 4, p. 939-943, 1996.

YODER, R. N. et al. Aerobic metabolism of diclosulam on U.S. and South American soils. J. Agric. Food Chem., v. 48, n. 1, p. $4335-4340,2000$

ZAR, H. J. Biostatistical analysis. New Jersey: Prentice Hall, 1999. p. 663. 